\title{
Rupture of Multiple Hepatic Artery Microaneurysms in Microscopic Polyangiitis
}

TAIICHIRO SHIRAI ${ }^{\circ}$, MD; TAKAICHI OKANO, MD, PhD; AKIO MORINOBU, MD, PhD, Department of Rheumatology and Clinical Immunology, Kobe University Hospital, Kobe, Japan. Address correspondence to Dr. T. Shirai, Department of Rheumatology and Clinical Immunology, Kobe University Hospital, 7-5-2, Kusunoki-cho, Chuo-ku, Kobe, 650-0017, Japan. E-mail: shirai@med.kobe-u.ac.jp. Ethics approval was not required in accordance with the policies of the authors' institution. Patient consent was received and is on file with the authors. J Rheumatol 2018;45:1705; doi:10.3899/jrheum.180276

Formation of hepatic artery aneurysms is very rare in microscopic polyangiitis (MPA), but the rupture of aneurysms can be fatal. We present a case of ruptured multiple hepatic artery microaneurysms in MPA successfully treated with rituximab (RTX).

An 89-year-old woman presented with a 3-week history of fever and numbness in the lower limbs. Laboratory tests revealed elevated levels of C-reactive protein, creatinine, myeloperoxidase antineutrophil cytoplasmic antibody (130 $\mathrm{U} / \mathrm{ml}$ ), and presence of microhematuria. Lower limb nerve conduction studies revealed mononeuritis multiplex. MPA was diagnosed. After admission, the patient suddenly complained of headache, hemoptysis, and severe abdominal pain. Head and chest computed tomography (CT) revealed putaminal hemorrhages and diffuse alveolar hemorrhage, respectively. Contrast-enhanced abdominal CT revealed hepatic hemorrhage in segment 4 (Figure 1A). Hepatic angiography disclosed extravasation from the arterial branch of segment 4 and multiple microaneurysms (Figure 1B). Hepatic arterial embolization was successfully performed, and remission induction was achieved by RTX combined with glucocorticoids.
Formation of hepatic artery aneurysms is very rare in MPA and is more typically presented by polyarteritis nodosa. In our patient, MPA was carefully diagnosed based on the European Medicines Agency algorithm ${ }^{1}$. Only 2 patients with hepatic artery aneurysms in MPA have been reported to date $^{2,3}$, one of whom died because of ruptures ${ }^{3}$. Notably, the rupture of hepatic artery aneurysms in MPA is rare but lethal, and remission induction with RTX is effective in such cases.

\section{REFERENCES}

1. Watts R, Lane S, Hanslik T, Hauser T, Hellmich B, Mahr A, et al. Development and validation of a consensus methodology for the classification of the ANCA-associated vasculitides and polyarteritis nodosa for epidemiological studies. Ann Rheum Dis 2007; 66:222-7.

2. Tamei N, Sugiura H, Takei T, Itabashi M, Uchida K, Nitta K. Ruptured arterial aneurysm of the kidney in a patient with microscopic polyangiitis. Intern Med 2008;47:521-6.

3. Guillevin L, Durand-Gasselin B, Cevallos R, Gayraud M, Lhote F, Callard P, et al. Microscopic polyangiitis: clinical and laboratory findings in eighty-five patients. Arthritis Rheum 1999;42:421-30.
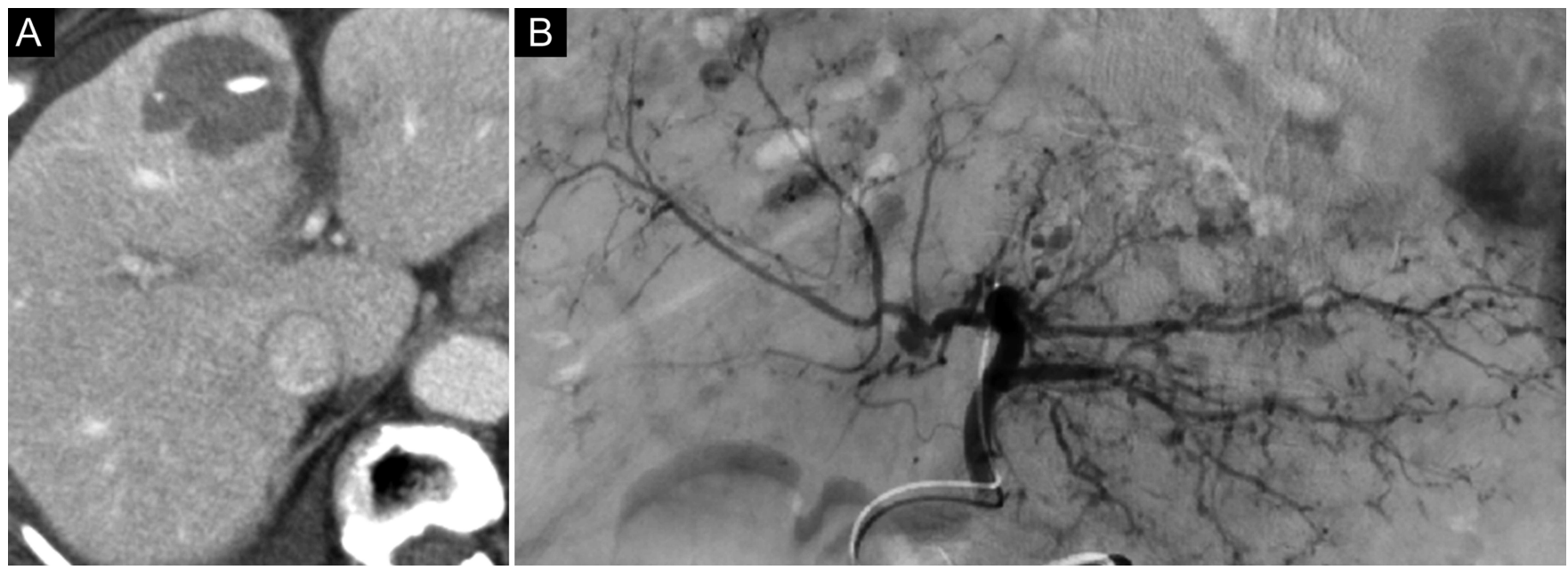

Figure 1. A. Contrast-enhanced abdominal CT showed hepatic hemorrhage in segment 4. B. Hepatic angiography disclosed extravasation from the arterial branch of segment 4 and multiple microaneurysms. CT: computed tomography.

Personal non-commercial use only. The Journal of Rheumatology Copyright $\odot$ 2018. All rights reserved 